\title{
Neurocognitive robot-assisted rehabilitation of hand function: a randomized control trial on motor recovery in subacute stroke
}

Raffaele Ranzani ${ }^{1 *}$ (D), Olivier Lambercy ${ }^{1 \dagger}$, Jean-Claude Metzger ${ }^{1}$, Antonella Califfi ${ }^{2}$, Stefania Regazzi ${ }^{2}$, Daria Dinacci ${ }^{2}$, Claudio Petrillo ${ }^{2}$, Paolo Rossi ${ }^{2}$, Fabio M. Conti ${ }^{2}$ and Roger Gassert ${ }^{1}$

\begin{abstract}
Background: Hand function is often impaired after stroke, strongly affecting the ability to perform daily activities. Upper limb robotic devices have been developed to complement rehabilitation therapy offered to persons who suffered a stroke, but they rarely focus on the training of hand sensorimotor function. The primary goal of this study was to evaluate whether robot-assisted therapy of hand function following a neurocognitive approach (i.e., combining motor training with somatosensory and cognitive tasks) produces an equivalent decrease in upper limb motor impairment compared to dose-matched conventional neurocognitive therapy, when embedded in the rehabilitation program of inpatients in the subacute stage after stroke.

Methods: A parallel-group, randomized controlled trial was conducted on subjects with subacute stroke receiving either conventional or robot-assisted neurocognitive hand therapy using a haptic device. Therapy was provided for 15, 45-min sessions over four weeks, nested within the standard therapy program. Primary outcome was the change from baseline in the upper extremity part of the Fugl-Meyer Assessment (FMA-UE) after the intervention, which was compared between groups using equivalence testing. Secondary outcome measures included upper limb motor, sensory and cognitive assessments, delivered therapy dose, as well as questionnaires on user technology acceptance.
\end{abstract}

Results: Thirty-three participants with stroke were enrolled. 14 subjects in the robot-assisted and 13 subjects in the conventional therapy group completed the study. At the end of intervention, week 8 and week 32, the robotassisted/conventional therapy group improved by 7.14/6.85, 7.79/7.31, and 8.64/8.08 points on the FMA-UE, respectively, establishing that motor recovery in the robot-assisted group is non-inferior to that in the control group.

\footnotetext{
* Correspondence: relab.publications@hest.ethz.ch

${ }^{\dagger}$ Raffaele Ranzani and Olivier Lambercy contributed equally to this work. ${ }^{1}$ Rehabilitation Engineering Laboratory, Institute of Robotics and Intelligent Systems, Department of Health Sciences and Technology, BAA Lengghalde 5, 8008 Zurich, Switzerland

Full list of author information is available at the end of the article
}

(c) The Author(s). 2020 Open Access This article is licensed under a Creative Commons Attribution 4.0 International License, which permits use, sharing, adaptation, distribution and reproduction in any medium or format, as long as you give appropriate credit to the original author(s) and the source, provide a link to the Creative Commons licence, and indicate if changes were made. The images or other third party material in this article are included in the article's Creative Commons licence, unless indicated otherwise in a credit line to the material. If material is not included in the article's Creative Commons licence and your intended use is not permitted by statutory regulation or exceeds the permitted use, you will need to obtain permission directly from the copyright holder. To view a copy of this licence, visit http://creativecommons.org/licenses/by/4.0/. The Creative Commons Public Domain Dedication waiver (http://creativecommons.org/publicdomain/zero/1.0/) applies to the data made available in this article, unless otherwise stated in a credit line to the data. 


\begin{abstract}
(Continued from previous page)
Conclusions: Neurocognitive robot-assisted therapy of hand function allows for a non-inferior motor recovery compared to conventional dose-matched neurocognitive therapy when performed during inpatient rehabilitation in the subacute stage. This allows the early familiarization of subjects with stroke to the use of such technologies, as a first step towards minimal therapist supervision in the clinic, or directly at home after hospital discharge, to help increase the dose of hand therapy for persons with stroke.
\end{abstract}

Trial registration: EUDAMED database (CIV-13-02-009921), clinicaltrials.gov (NCT02096445). Registered 26 March 2014 - Retrospectively registered, https://linicaltrials.gov/ct2/show/NCT02096445

Keywords: Neurorehabilitation, Stroke, Hand, Robotics, Haptics

\section{Background}

Upper-limb robot-assisted therapy has been established as a safe and feasible treatment to complement rehabilitation after neurological injury, such as stroke [1]. Robots can precisely control the interaction with the user (e.g., supporting or resisting in an assist-as-needed manner) and render virtual environments both visually and mechanically, making them ideal tools for sensorimotor training, providing engaging and challenging therapy $[2$, $3]$. Over the past two decades, several robotic devices to train the proximal upper extremity [4] were developed and clinically evaluated, achieving outcomes comparable to dose-matched conventional therapy $[1-3,5-10]$.

However, distal arm function is essential for the execution of activities of daily living (e.g., eating, dressing) and is often severely impaired after stroke [11], with low probability of regaining its full functional use [12]. Several studies have shown that functional motor training at the level of the hand with robotic devices can be beneficial and positively translate into recovery of proximal arm function [13, 14]. Despite recent investigations to develop novel robots to train hand function $[9,15$, 16], only few systems took advantage of the haptic rendering capabilities of robots to support somatosensory training, nor evaluated this in clinical trials. As such, most systems for robot-assisted therapy developed to date focus on movement practice without incorporating an established therapy concept adapted to the capabilities of the respective technology.

In this work, the clinical equivalence of sensorimotor, robot-assisted rehabilitation of hand function is investigated within a four-week randomized controlled trial (RCT) on subacute stroke participants. The neurocognitive rehabilitation method proposed by Perfetti [17] was selected as reference therapy approach. It focuses on the training of sensorimotor functions as well as cognition, which is fundamental during functional interactions between body and environment (e.g., information perception, as well as elaboration, selection and execution of motor plans) [18-20]. Because of the relevance of the cognitive processing of sensory inputs, this approach is particularly interesting for hand rehabilitation. Moreover, the integration of multisensory inputs promotes the involvement of associative cortices that play a key role in learning and consequently in neuronal plasticity and recovery [21]. While only a few studies compared neurocognitive therapy to other rehabilitative approaches [18, 22], some promising work suggested that it can significantly improve upper-limb function, ability to perform activities of daily living and quality of life compared to conventional task-oriented training [22]. Consequently, this approach has recently found increasing interest in the scientific community, applied both in conventional [23-25] and in technology-assisted therapy $[26,27]$, but has so far not been evaluated in the context of a robot-assisted RCT. The therapy concept inspired by the neurocognitive approach was implemented on a high-fidelity 2 degrees of freedom endeffector haptic device to train hand function (i.e., the ReHapticKnob [28]). The therapy exercises focused on grasping and pronosupination (e.g., tactile discrimination tasks, teach and reproduce tasks, haptic exploration tasks, [29]) and were performed using virtual objects rendered both visually and haptically by the robot, mimicking the physical objects used in conventional therapy. The primary objective of this RCT was to investigate if the implemented robot-assisted hand therapy concept could be integrated into the rehabilitation program of participants with subacute stroke during their inpatient stay (i.e., replace one conventional neurocognitive therapy session on each intervention day) and if, at precisely matched dose, an equivalent reduction in upper limb motor impairment could be achieved. This study design was motivated by the need to establish noninferiority in terms of rehabilitation outcomes when comparing the proposed intervention to conventional neurocognitive therapy. This is an important first step towards the investigation of more specific robot-assisted protocols that could further take advantage of the abilities of the robotic device, such as increasing dose through semi-supervised therapy. As secondary objectives, we hypothesized that neurocognitive robot-assisted 
therapy of the hand would lead to improvements in motor, sensory and cognitive functions in participants with subacute stroke.

\section{Methods}

\section{Trial design}

A single center, parallel group, randomized control trial was conducted at the Clinica Hildebrand Centro di Riabilitazione Brissago, Switzerland. Study participants were recruited among inpatients undergoing an intensive interdisciplinary rehabilitation therapy program poststroke. After screening for eligibility by a medical doctor, participants were randomly assigned (by balanced prerandomization [1:1]) to a robot-assisted group (RG), receiving robot-assisted neurocognitive therapy with the ReHapticKnob (see Fig. 1) haptic device, or to a control group (CG), receiving dose-matched conventional neurocognitive therapy without the robot. On 15 days

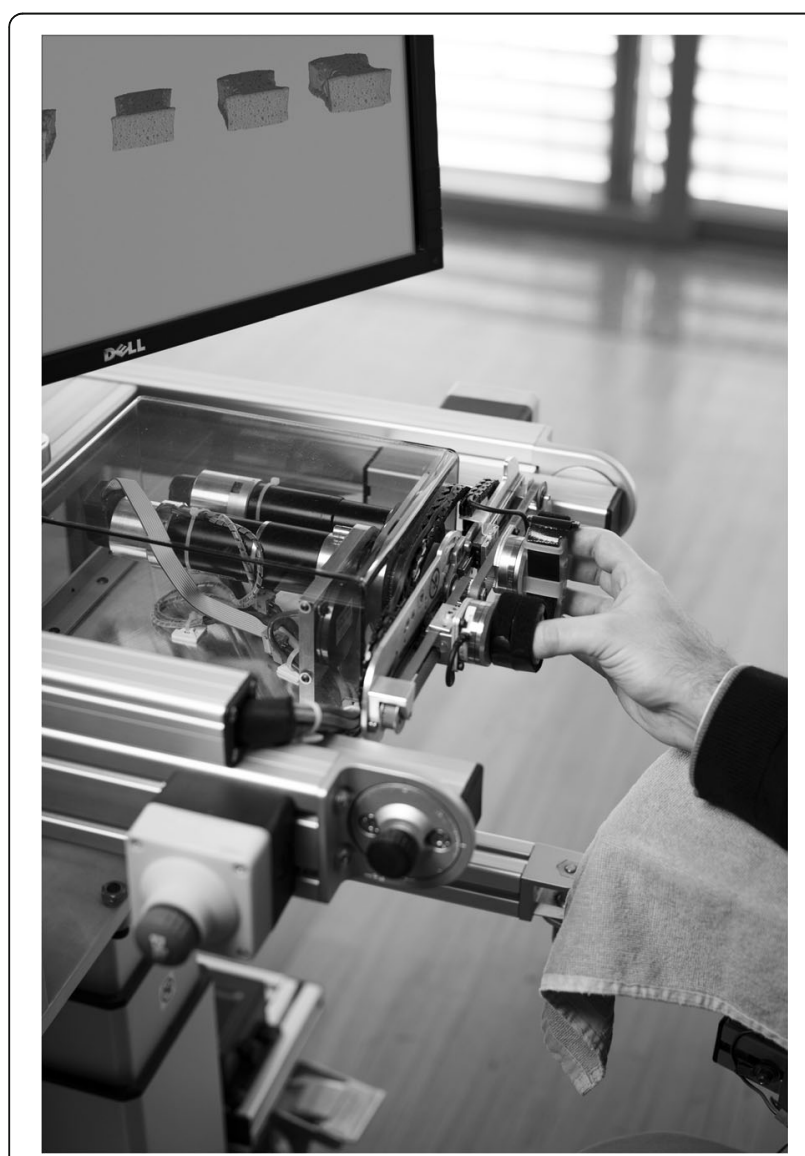

Fig. 1 A subject with stroke using the ReHapticKnob. The ReHapticknob is a haptic device used to train hand opening-closing and forearm pronosupination. The device integrates a set of 7 therapy exercises reproducing typical neurocognitive exercises [29]. In the present exercise, the compliance of different virtual sponges rendered by the device has to be memorized and identified by relying on hand somatosensory inputs during active interaction with the device distributed over 4 weeks, all subjects received three neurocognitive therapy sessions (i.e., $2 \times 45 \mathrm{~min}$ and $1 \times$ $30 \mathrm{~min}$ ) per day focusing on hand function (see Fig. 2). In the RG, one of the 45 min therapy sessions per day was substituted with robot-assisted therapy. Based on ethical grounds, only one session of upper limb therapy per day was replaced to guarantee that all patients could still get access to the standard treatment for subacute inpatients. These sessions were embedded in the weekly therapy plan of each individual participant. The study protocol was reviewed and approved by the local Ethics Committee (EC 2646) and Swissmedic (2013-MD-0002) prior to participant recruitment. Simultaneously, the study was registered on the (non-public) European register EUDAMED and subsequently in Clinialtrials.gov.

\section{Participants}

Subjects were enrolled in the study if they met the following inclusion criteria: age between 18 and 90 years old, first and only cerebrovascular event, subacute lesion (i.e., occurred not earlier than 6 weeks before recruitment), hemiparesis with arm motor deficit as assessed with a National Institutes of Health Stroke Scale (NIHS $S$, [30]) $\geq 1$. Subjects were excluded if they presented an altered state of consciousness, severe aphasia (Goodglass and Kaplan test $<1$, [31]), severe cognitive deficits (Levels of Cognitive Functioning-Revised, LCF-R $<6$, [32]), severe pathologies of the upper limb of traumatic or rheumatic nature, severe pain in the affected arm $(\geq 5$ on a visual analogue scale for pain (VASp)), or if they had active pacemakers and other active implants.

\section{Interventions}

The neurocognitive therapy approach proposed by Perfetti [17] includes sensorimotor and cognitive aspects, all fundamental during the execution of complex tasks and activities of daily life. Focusing on haptic and postural perception, often without vision, subjects are asked to explore objects (e.g. sponges, sticks, springs), discriminate their properties and perceive relative differences. A robotic device is an ideal tool to perform such exercises, as a wide range of haptic stimuli can easily and accurately be rendered in a repeatable and well-controlled manner [29]. Seven exercises were available both in conventional and robot-assisted therapy: passive grip aperture discrimination, passive pronosupination angle identification, stiffness identification during grasping, stiffness identification during index finger pinching, teach and reproduce of grip apertures, teach and reproduce of pronosupination angles. The seventh exercise in the conventional therapy was a texture identification exercise, while in the robot-assisted therapy, the exercise consisted in the identification of specific pronosupination angles, indicated by a vibratory cue on the grasping 


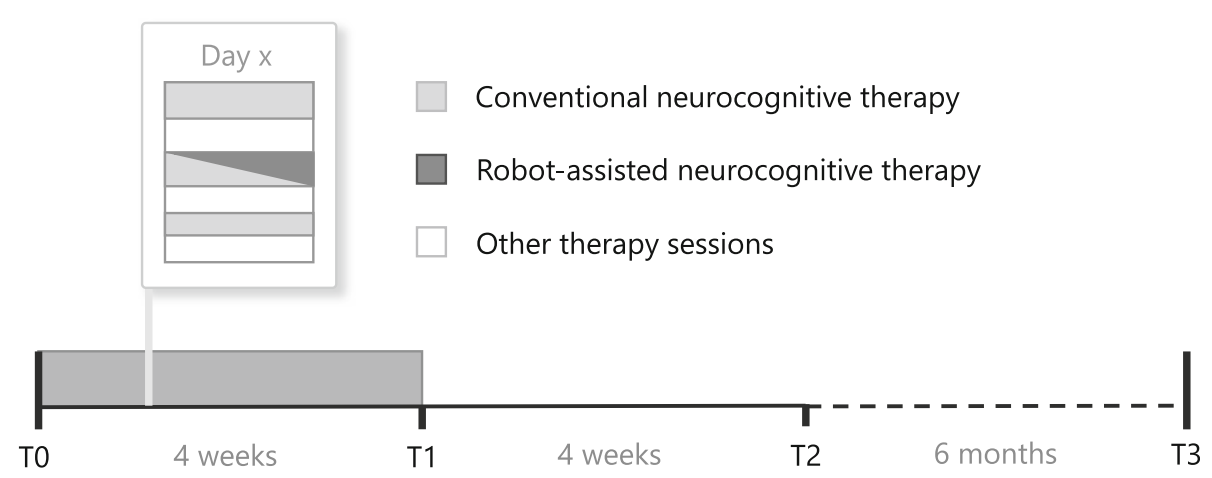

Fig. 2 Study protocol. Integration of RCT therapy sessions into the weekly therapy schedule of participants and assessment scheduling. Assessment sessions were performed at therapy start (T0), after the 4-week intervention (T1), as well as at 4-week (T2) and 6-month (T3) follow-ups

DOF (within a $4^{\circ}$ window around the targeted pronosupination angle). Within these exercises, the motor aspects of the intervention consisted of symmetric thumb and fingers flexion/extension, as well as forearm pronation/supination, which were executed either independently or combined. The sensory aspects of the intervention entailed encoding (i.e., perception and processing) the following types of somatosensory signals without visual information: sponge/spring stiffnesses, size and shape of objects (e.g. stick lengths, sponge size), arm positioning (e.g., pronosupination orientations), and vibratory cues. The cognitive aspects of the training demanded elaboration/recognition of perceptual information (e.g., understand and memorize object length/ stiffness), encoding/decoding of this information in the working memory for comparison purposes of more than one object (e.g., identify length/stiffness of an unknown object), planning/execution/correction of fine motor plans. The tasks were executed either passively (i.e., guided by the therapist/robot) when they only required sensory perception (e.g. of object length or forearm orientation), or actively by the subject (against the resistance of the object/robot) when they required active object manipulation (e.g., stiffness identification). The robotic device used in this study can haptically reproduce the same objects and, thereby, motor, sensory and cognitive tasks used in conventional therapy. The objects are rendered via the robotic handles by generating appropriate forces during hand opening/closing and forearm pronosupination, while they are displayed on a screen (see Fig. 1) [28].

In both groups, all the conventional neurocognitive therapy sessions included two or three exercises depending on the session duration (i.e., 30 or $45 \mathrm{~min}$ ), as typically done in the standard clinical setting. The exercises were performed with the help of the therapist, who progressively adapted the assistance and difficulty level of the exercise (e.g., number of objects, object length or stiffness) depending on his/her evaluation of the subject's ability.

Similarly, each 45-min session of robot-assisted therapy included three exercises (selected each day following a predefined plan common to all participants) consisting of up to 30 task repetitions with the robot (each involving multiple movements and interpretation of sensory information), in a maximum of $15 \mathrm{~min}$ per exercise. The exercise type, number of task repetitions per exercise and the maximum exercise duration were selected based on pilot tests on subjects with stroke [29] to precisely match therapy type and dose typically performed in conventional therapy. In each exercise, the difficulty level was initially adapted to the subject according to a baseline robotic assessment and continuously updated at the end of each session depending on the subject's performance. An experienced physio- or occupational therapist supervised all the sessions. For a more detailed description of the robotic assessments, exercises and difficulty adaptation, refer to our earlier work [29].

\section{Outcome measures and masking}

Participants were evaluated on separate days with respect to the therapy sessions, at four time points: before (T0) and after (T1) the intervention, and in two follow-ups at 8 weeks (T2) and 32 weeks (T3) (see Fig. 2). Assessors were masked to treatment allocation, while participants, therapists and data analysts were unmasked.

\section{Primary outcome}

The primary outcome of the study, which was tested for equivalence, was the change from baseline in upper extremity motor impairment at the end of treatment (i.e., T1-T0), assessed with the Fugl-Meyer Assessment of the Upper Extremity (FMA-UE) [33]. The FMA-UE scale was chosen as primary outcome due to its relevance in 
sensorimotor rehabilitation and related literature, especially with respect to robot-assisted therapy.

\section{Secondary outcomes}

The secondary outcomes of the study are divided into three categories to compare the two intervention groups at each time point:

1. Motor, sensory and cognitive scales: changes in upper limb impairment at each time point were measured using the FMA-UE and its subcomponents related to hand and wrist (FMA-WH) as well as shoulder and elbow (FMA-SE), gross manual dexterity using the Box and Block Test (BBT) [34], spasticity level of the upper limb (i.e., shoulder adductors, elbow flexors and extensors, wrist flexors and finger flexors) with the Modified Ashworth Scale (MAS) [35], tactile sensation and proprioceptive ability of the upper limb with the Erasmus MC Nottingham Sensory Assessment (EmNSA) [36], cognitive impairment with the Mini Mental State Examination (MMSE) [37], unilateral spatial neglect with the Albert Test (AT) [38], and behavioral ability and dementia with the Frontal Assessment Battery (FAB) [39].

2. Therapy intensity: to verify dose matching, the two groups were compared in terms of average number of task repetitions performed in one session and therapy intensity (i.e., number of task repetitions per minute of effective therapy). During conventional therapy, the number of task repetitions and the effective therapy time were recorded by the supervising therapist, while they were directly logged by the robot during the robotassisted therapy.

3. Acceptance of neurocognitive robot-assisted therapy: in the subjects from the RG, acceptance was evaluated by a subjective 4-item questionnaire: (Q1) "Are the exercises with the robot motivating?" (0 no, 1 yes), (Q2) "Would you recommend the additional robot therapy to other subjects with stroke?" (0 no, 1 yes), (Q3) “Did the robot-therapy lead to concrete improvements?" (0 no, 1 yes), (Q4) "How comfortable were the exercises with the robot for you?" (0 uncomfortable, 10 very comfortable).

\section{Statistical methods and sample calculation}

The Wilcoxon Rank Sum Test was used to assess homogeneity between groups at baseline for time post lesion, FMA-UE, FMA-WH, NIHSS, LCF-R and GoodglassKaplan, and a two-sample t-test for age and VASp, which resulted to be normally distributed. Fisher's exact test was applied to investigate group differences in gender, side of stroke, and stroke type. Measurements of the average dose and therapy intensity in RG and CG were also compared using the two-sample t-test or the Wilcoxon Rank Sum Test.

Equivalence testing [40] was used to investigate whether the groups showed an equivalent change in terms of the primary outcome measure. Equivalence was established if the difference in change in FMA-UE between the two groups lies within an equivalence boundary of \pm 5.2 points, which was reported to be the minimal detectable change for the FMA-UE [41]. The confidence intervals were calculated as described by D'Agostino et al. for small sample sizes $(<30)$ [42]. The equivalence test was repeated at T1 (primary outcome), $\mathrm{T} 2$ and $\mathrm{T} 3$ to evaluate if equivalence is retained over time. A pre-study power calculation for equivalence testing estimated that 28 subjects would provide $80 \%$ power to prove equivalence between the two groups in terms of FMA-UE, given the selected equivalence boundary and an estimated standard deviation of 4.66 FMA-UE points in the FMA-UE score change after therapy (based on preliminary data [29]). To compensate for an expected dropout rate of $15 \%$, a sample size of 32 participants was selected.

For all outcome measures, the groups were compared after the intervention (T1-T0) and at the follow-ups (T2-T0 and T3-T0) in a $2 \times 3$ (i.e., group $x$ time) repeated measures analysis of variance (ANOVA) analyzing between and within-group differences. In presence of significant differences, post-hoc comparisons were performed between T1-T0, T2-T0 and T3-T0. The statistical significance level of $\alpha=0.05$ was corrected using Bonferroni correction in the analyses of the primary and secondary outcome measures (i.e., ANOVA), leading to a value of 0.0046 and 0.0025 , respectively.

To obtain a meaningful estimate of the treatment effect, all analyses were performed by modified intention to treat (MITT): all assigned participants for whom outcome data at the end of the intervention (T1) are available were analyzed. For missing data, we inferred the missing value by last observation carried forward or, if no former value was available, by next observation carried backward.

\section{Results}

Between April 2013 and March 2017, 33 subjects with subacute stroke were eligible and agreed to participate in the study (Fig. 3). We did not keep a complete log of subjects who were screened for eligibility, but this number was estimated to be between 80 and 90 by the principal investigator. The target sample size was reached with 17 subjects allocated to the RG and 16 subjects allocated to the CG. Only 27 subjects received the allocated intervention and completed the $\mathrm{T} 1$ assessment (MITT population: 14 RG, 13 CG), six subjects did not 


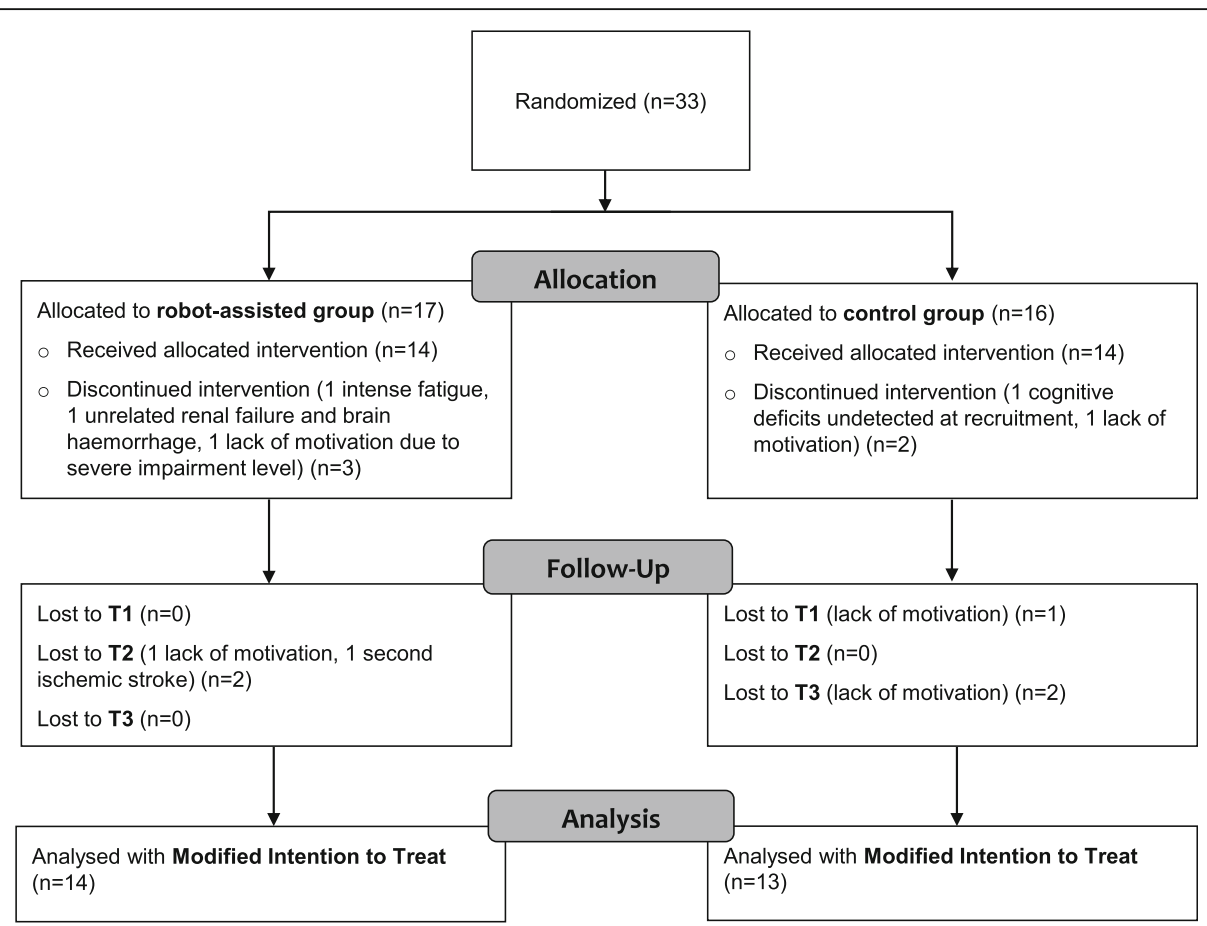

Fig. 3 Trial profile describing the participants population of the RCT

complete the intervention protocol or withdrew before the T1 assessment due to lack of motivation, concomitant unrelated medical pathologies or cognitive deficits that were not detected at recruitment. Twenty-three subjects (12 RG, $11 \mathrm{CG}$ ) completed the full protocol up to $\mathrm{T} 3$ as 1 subjects had a recurrent stroke and 3 additional subjects withdrew due to a lack of motivation after the completion of the intervention. During the duration of the study, no adverse event related to the intervention was observed.

\section{Baseline characteristics}

Table 1 reports the baseline demographics and clinical characteristics of the two groups at T0. No statistically significant differences were found in baseline characteristics (Wilcoxon Rank Sum Test, two-sample t-test, Fisher's exact test, see Table 1 for more details). The participant age range was 38 to 85 years and there were 12 right and 15 left hemisphere lesions. Most subjects showed mild/moderate [43] initial upper-limb impairment (FMA-UE $50.48 \pm 13.50$ (mean \pm std)) due to both ischemic and hemorrhagic stroke. In the two groups, a different distribution in stroke type was evident (although not significantly different after Bonferroni correction), with a majority of ischemic strokes in the robot-assisted group. Before enrollment, all participants were informed about the study and gave written consent.

\section{Equivalence in Fugl-Meyer of the upper extremity}

According to the equivalence analysis (Fig. 4), the change in FMA-UE in the robot-assisted group can be considered as non-inferior to the control group. The 90\% confidence interval lies within the equivalence boundaries at $\mathrm{T} 1$ (i.e., primary outcome) but tends to move outside the equivalence boundary in favor of the robot-assisted therapy at the end of the study (T3). Between $\mathrm{T} 0$ and $\mathrm{T} 1$, subjects in the RG improved on average by 7.14 FMA points, while those in the CG showed an average increase of 6.85 FMA points. In both groups, these changes are above the minimal detectable/ clinically important change (i.e., 5.2 and 5.25 FMA-UE points, respectively) [41, 44].

The changes in FMA-UE were maintained at T2 and T3 (i.e., secondary outcome measures). Between T0 and $\mathrm{T} 2$, subjects in the RG improved on average by 7.79 FMA points, while those in the CG showed an average increase of 7.31 FMA points. Finally, from T0 to T3, RG subjects improved by 8.64 FMA points and CG subjects by 8.08 FMA points.

\section{Changes in secondary outcome measures}

Comparing the changes in clinical scales with respect to baseline over time (see Table 2), the two groups did not show any significant between-group difference, as shown by the group factor in ANOVA. Additionally, therapyinduced T1-T0 within-group changes of each scale were 
Table 1 Baseline characteristics of the randomized study participants (MITT, $N=27$ )

\begin{tabular}{|c|c|c|c|}
\hline Characteristics $^{a}$ & Robot-assisted $(n=14)$ & Control $(n=13)$ & $p^{b}$ \\
\hline Age (years) & $70.00(12.79)$ & $67.46(11.39)$ & $0.5921(t(25)=0.543)$ \\
\hline Time since stroke (weeks) & $3.14(1.51)$ & $3.08(1.32)$ & $0.8794(Z=-0.2)$ \\
\hline Sex & & & 0.6946 \\
\hline Male & 10 & 8 & \\
\hline Female & 4 & 5 & \\
\hline Side of stroke & & & 1.000 \\
\hline Left & 8 & 7 & \\
\hline Right & 6 & 6 & \\
\hline Stroke type & & & 0.0054 \\
\hline Ischemic & 13 & 5 & \\
\hline Hemorrhagic & 1 & 7 & \\
\hline Both & 0 & 1 & \\
\hline FMA-UE & $50.14(12.50)$ & $50.84(15.01)$ & $0.7891(Z=-0.3)$ \\
\hline FMA-WH & $17.86(5.61)$ & $19.39(6.20)$ & $0.1478(Z=-1.4)$ \\
\hline NIHSS & $1.36(0.75)$ & $1.69(1.03)$ & $0.3500(Z=-0.9)$ \\
\hline VASp & $0.00(0.00)$ & $0.85(1.63)$ & $0.0623(t(25)=-1.951)$ \\
\hline LCF-R & $8.86(1.10)$ & $8.31(1.44)$ & $0.3390(Z=1.0)$ \\
\hline Goodglass-Kaplan & $4.43(0.76)$ & $4.31(1.18)$ & $0.8438(Z=-0.2)$ \\
\hline
\end{tabular}

Abbreviations: FMA-UE Fugl-Meyer Assessment of the Upper Extremity; FMA-WH Fugl-Meyer Assessment of Wrist and Hand functions; NIHSS National Institutes of Health Stroke Scale; VASp Visual Analogue Scale for Pain; LCF-R Revised Levels of Cognitive Function; Goodglass-Kaplan, Assessment of aphasia and related disorders

${ }^{a}$ Continuous data are expressed as mean (standard deviation), categorical data as number

${ }^{\mathrm{b}} p$ values are associated with the Fisher's exact test for categorical variables (used for small samples), while Wilcoxon rank sum test and two-sample t-test are used for continuous variables (independent samples). According to the Bonferroni correction, the significance level $\boldsymbol{a}^{*}=\frac{\mathbf{0 . 0 5}}{\mathbf{1 1}}=\mathbf{0 . 0 0 4 5 5}$ )

maintained at $\mathrm{T} 2$ and $\mathrm{T} 3$, as shown by the time factor in the ANOVA analysis, except for the BBT score that continued to increase. At T1, the FMA-WH improved by 2.93 and 2.39 FMA points in the RG and CG, respectively, while the FMA-SE improved by 4.21 FMA points in the RG and 4.46 FMA points in the CG. BBT increased by 11.43 blocks/min on average in the RG, and 12.85 blocks/min in the CG. As for the FMA-UE, the BBT change at T1-T0 was above its minimal detectable change of 5.5 blocks/min [45] in both groups. A significant time dependency after the end of therapy was observed, and post-hoc analysis suggested an increase of BBT between T1 and T3 $(\mathrm{t}(52)=-2.396, p=0.020)$, although this change was not significant following Bonferroni correction. The EmNSA-T increased by 1.07 points in the RG and 2.85 points in the CG, while the EmNMA-P increased by 0.14 and 0.54 points, respectively. The MAS showed a negligible increase of 0.07 points in the RG and decreased by 1.54 points in the CG at $\mathrm{T} 1$, converging to the same score range (i.e., around 1 MAS point) at T3. A T1-T0 decrease above the MAS minimal detectable change of 1 point [46] was only detected in the CG, which could be explained by the slightly higher MAS baseline score of this group. From T0 to T3, the MAS decreased by 0.29 and 0.85 points in the RG and CG, respectively. The MMSE increased by 0.57 and 1.05 points in the RG and CG, respectively, but both changes were below the MMSE minimal detectable change of 3 points [47]. The FAB increased by 0.43 and 1.26 points in the RG and CG, respectively, while the Albert Test showed minor increases of 0.07 and 0.15 points.

\section{Therapy intensity}

During a therapy session, the RG performed on average $71.49 \pm 10.84$ task repetitions while the CG received $73.47 \pm 45.19$ task repetitions, as reported by the supervising therapist. The average number of task repetitions per session received in the RG and in the CG was not significantly different as revealed by the twosample $\mathrm{t}$-test $(\mathrm{t}(23)=-0.159, p=0.875)$. In terms of therapy intensity, there was also no statistically significant difference between the two groups, either comparing robot-assisted and conventional therapy sessions $(\mathrm{RG}=1.45 \pm 0.33 \mathrm{reps} / \mathrm{min}, C G=1.40 \pm 0.81 \mathrm{reps} / \mathrm{min}$, Wilcoxon Rank Sum Test $\mathrm{Z}=-0.8, p=0.427$ ) or comparing all conventional therapy sessions in both groups $(\mathrm{RG}=1.63 \pm 0.85 \mathrm{reps} / \mathrm{min}, \quad \mathrm{CG}=1.40 \pm 0.81 \mathrm{reps} / \mathrm{min}$, Wilcoxon Rank Sum Test $\mathrm{Z}=-0.6, p=0.529)$. In addition to the neurocognitive therapy sessions, the 

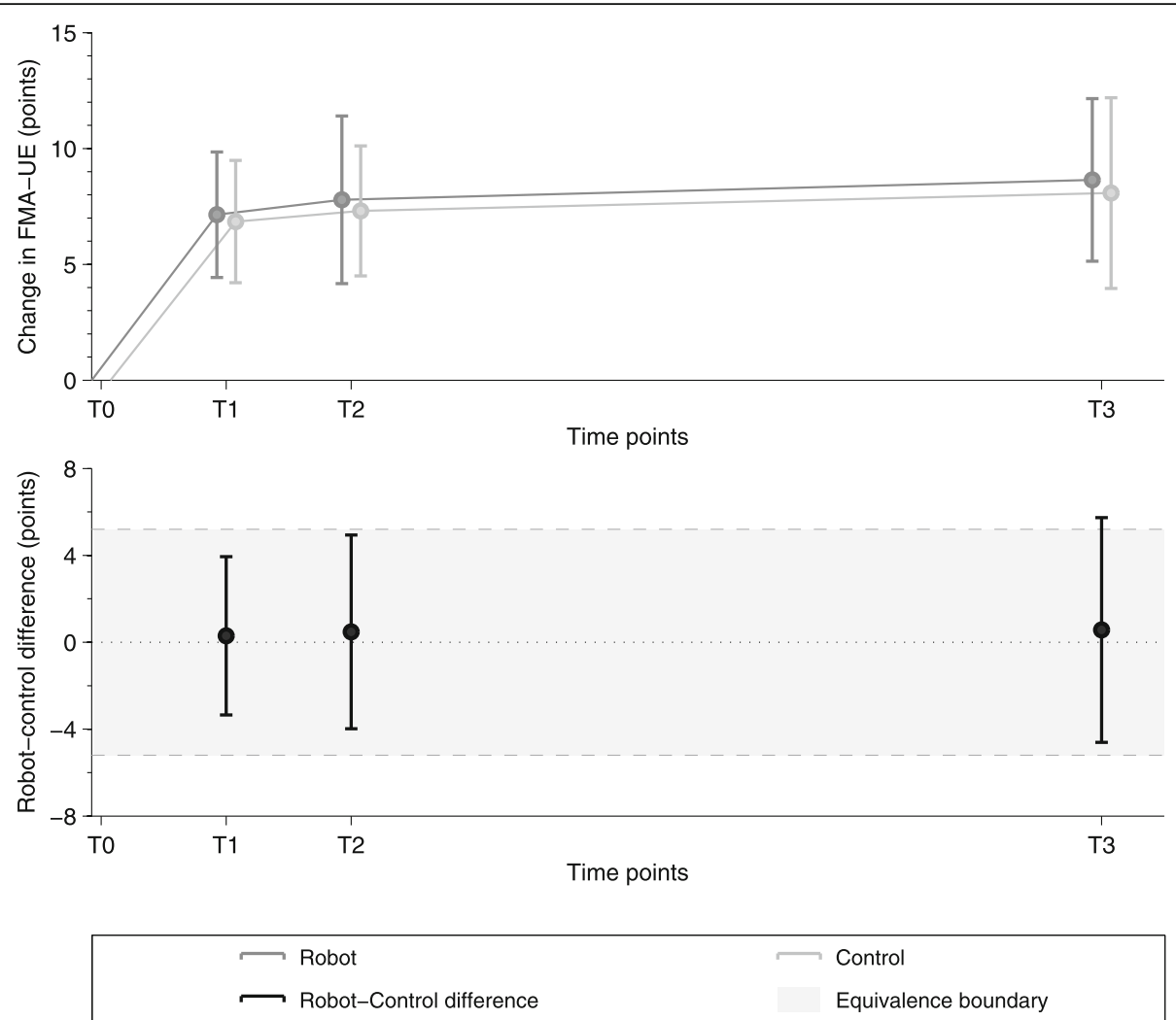

Fig. 4 Equivalence test between robot-assisted and control group w.r.t. the FMA-UE change. The test was performed at 4 weeks (T1), 8 weeks (T2) and 8 months (T3) compared to baseline

average daily amount of occupational therapy and/or lower limb physiotherapy did not statistically differ in the two groups $(\mathrm{RG}=40.68 \pm 17.88 \mathrm{~min}, \mathrm{CG}=50.33 \pm$ $6.41 \mathrm{~min})$, as revealed by the two-sample t-test $(\mathrm{t}(23)=-1.699, p=0.103)$.

\section{Acceptance of neurocognitive robot-assisted therapy}

Out of 12 participants that answered the questionnaire in the RG, $91.7 \%$ found the robot-assisted therapy motivating (Q1), 84.6\% would recommend the robotassisted therapy program to other persons with stroke (Q2), and $84.6 \%$ found concrete improvements in their health status at the end of the therapy program (Q3). Participants found the robot-assisted therapy to be comfortable, rating it at $7.42 \pm 1.34$ out of $10(\mathrm{Q} 4)$. The questionnaire revealed mild sporadic discomfort in the finger fixation, and that, in three out of seven exercises, difficulty levels were sometimes perceived as too high.

\section{Discussion}

This paper presents the clinical feasibility and outcomes of a RCT conducted on subjects with subacute stroke evaluating the effect of robot-assisted neurocognitive therapy of hand function, and in particular, if therapy with the haptic device could lead to an equivalent and lasting sensorimotor recovery compared to dose-matched conventional neurocognitive therapy. In contrast to most robot-assisted rehabilitation trials, which placed a strong focus on movement training, our approach takes full advantage of the haptic rendering abilities of the robot, and proposes a therapy program adapted to these capabilities. We could show that this approach is well accepted and recommended by the majority of the patients, and that it could be integrated in the daily schedule of inpatients in the subacute stage after stroke. Most participants found the program motivating, comfortable, and could perceive concrete improvements in their health status after the end of the treatment.

\section{Equivalent reduction in upper limb motor impairment}

Traditionally, most RCTs have aimed to prove that robot-assisted therapy per se could increase upper limb recovery with respect to conventional therapy (e.g., by increasing therapy intensity, subject engagement, or by providing exercises targeting specific motor impairments). However, large clinical studies on arm rehabilitation with subjects with chronic stroke, aiming to demonstrate the superiority of robot-assisted therapy, were rarely successful, or only observed small, nonclinically meaningful differences [2, 3]. Similarly, other 
Table 2 Patients' scores in all the clinical and robotic outcome measures

\begin{tabular}{|c|c|c|c|c|c|c|c|c|c|}
\hline \multirow{3}{*}{$\begin{array}{l}\text { Assessment } \\
\text { (Max/Healthy } \\
\text { Value) }\end{array}$} & \multirow[t]{3}{*}{ Group } & \multirow{3}{*}{$\begin{array}{l}\text { Baseline (TO) } \\
\text { value, Mean } \\
\text { (SD) }\end{array}$} & \multicolumn{3}{|c|}{ Change from baseline, Mean (SD) } & \multicolumn{4}{|c|}{ Repeated Measures ANOVA on change from baseline } \\
\hline & & & \multirow[t]{2}{*}{$\mathrm{T1}$} & \multirow[t]{2}{*}{$\mathrm{T} 2$} & \multirow[t]{2}{*}{ T3 } & \multicolumn{2}{|l|}{ Group } & \multicolumn{2}{|l|}{ Time } \\
\hline & & & & & & $F(1,25)$ & $P$ & $\mathrm{~F}(2,52)$ & $P$ \\
\hline \multirow[t]{2}{*}{ FMA-UE (66) } & RG & $50.14(12.50)$ & $+7.14(5.72)$ & $+7.79(7.65)$ & $+8.64(7.42)$ & 0.035 & 0.8537 & 1.206 & 0.3076 \\
\hline & CG & $50.85(15.00)$ & $+6.85(5.34)$ & $+7.31(5.68)$ & $+8.08(8.32)$ & & & & \\
\hline \multirow[t]{2}{*}{ FMA-WH (24) } & RG & $17.86(5.61)$ & $+2.93(2.62)$ & $+3.64(3.25)$ & $+3.64(3.23)$ & 0.371 & 0.5480 & 3.701 & 0.0314 \\
\hline & CG & $19.39(6.20)$ & $+2.39(1.81)$ & $+2.54(2.22)$ & $+3.39(3.62)$ & & & & \\
\hline \multirow[t]{2}{*}{ FMA-SE (42) } & RG & $32.29(8.08)$ & $+4.21(4.14)$ & $+4.14(5.92)$ & $+5.00(5.68)$ & 0.012 & 0.9152 & 0.303 & 0.7403 \\
\hline & $C G$ & $31.46(8.95)$ & $+4.46(3.91)$ & $+4.77(4.46)$ & $+4.69(5.62)$ & & & & \\
\hline \multirow[t]{2}{*}{ BBT (-) } & $R G$ & $17.79(9.67)$ & $+11.43(6.60)$ & $+13.50(7.33)$ & $+17.57(10.91)$ & 0.504 & 0.4842 & 11.330 & $8.241 e-05^{*}$ \\
\hline & CG & $15.15(8.44)$ & $+12.85(8.22)$ & + 17.54 (13.39) & + 19.92 (13.96) & & & & \\
\hline \multirow[t]{2}{*}{ EmNSA-T(24) } & $R G$ & $20.93(5.44)$ & + $1.07(2.20)$ & $+1.43(2.59)$ & $+1.86(3.33)$ & 2.986 & 0.0964 & 2651 & 0.0801 \\
\hline & CG & $15.15(9.44)$ & $+2.85(4.45)$ & $+5.54(7.33)$ & $+4.92(7.53)$ & & & & \\
\hline \multirow[t]{2}{*}{ EmNSA-P(8) } & $R G$ & $7.79(0.58)$ & $+0.14(0.36)$ & $-0.21(0.43)$ & $0.00(0.56)$ & 5.258 & 0.0305 & 0.520 & 0.5976 \\
\hline & CG & 6.77 (1.92) & $+0.54(0.97)$ & $+0.69(1.18)$ & $+0.46(0.78)$ & & & & \\
\hline \multirow[t]{2}{*}{$\operatorname{MAS}(0)$} & $R G$ & $1.29(1.77)$ & $+0.07(2.37)$ & $-0.21(2.36)$ & $-0.29(2.56)$ & 1.012 & 0.3241 & 0.558 & 0.5756 \\
\hline & CG & $2.15(2.94)$ & $-1.54(2.91)$ & $-1.31(3.12)$ & $-0.85(3.69)$ & & & & \\
\hline \multirow[t]{2}{*}{ MMSE(30) } & $\mathrm{RG}$ & $25.89(3.60)$ & $+0.57(1.91)$ & $+0.93(1.64)$ & $+1.71(3.07)$ & 0.072 & 0.7906 & 1.088 & 0.3446 \\
\hline & CG & $23.62(5.47)$ & $+1.05(1.87)$ & $+0.59(2.41)$ & $+0.93(3.33)$ & & & & \\
\hline \multirow[t]{2}{*}{$\mathrm{FAB}(18)$} & $\mathrm{RG}$ & $14.60(2.38)$ & $+0.43(1.74)$ & $+1.14(1.70)$ & $+1.61(1.67)$ & 0.144 & 0.7079 & 1.625 & 0.2067 \\
\hline & CG & $11.98(5.29)$ & $+1.26(1.71)$ & $+1.49(1.76)$ & $+1.05(1.60)$ & & & & \\
\hline \multirow[t]{2}{*}{ Albert Test (32) } & $R G$ & $31.86(0.36)$ & $+0.07(0.27)$ & $+0.07(0.48)$ & $+0.14(0.36)$ & 0.034 & 0.8544 & 0.000 & 1.000 \\
\hline & CG & $31.77(0.83)$ & $+0.15(0.90)$ & $+0.15(0.56)$ & $+0.08(0.28)$ & & & & \\
\hline
\end{tabular}

Abbreviations: SD Standard deviation; FMA-UE Fugl-Meyer Assessment of the Upper Extremity; FMA-WH Fugl-Meyer Assessment of Wrist and Hand functions; FMASE Fugl-Meyer Assessment of Shoulder and Elbow functions; MAS Modified Ashworth Scale; EmNSA-T Erasmus MC Nottingham Sensory Assessment of Tactile sensation; EmNSA-P Erasmus MC Nottingham Sensory Assessment of Tactile of Proprioceptive ability; MMSE Mini Mental State Examination; FAB Frontal Assessment Battery

Symbols: * Statistically significant according to Bonferroni correction (i.e., significance level $\boldsymbol{a}^{*}=\frac{\mathbf{0 . 0 5}}{\mathbf{2 0}}=\mathbf{0 . 0 0 2 5}$ )

studies focusing solely on robot-assisted rehabilitation of hand function in chronic [48-51] or subacute stroke [12, $52,53]$ were not able to show statistically significant differences between robot and control therapy groups, or reported minor differences in secondary outcome measures $[10,54,55]$. The present RCT directly investigated equivalence in motor impairment reduction between a robot-assisted and a conventional therapy group focusing on the training of the upper limb, and in particular the hand. For this purpose, the therapy dose (i.e., total number of task repetitions and therapy time) as well as the therapy intensity (i.e., task repetitions per time unit) were precisely matched between groups.

The results of the equivalence test comparing the evolution in FMA-UE demonstrate that, for our specific intervention, the motor recovery in the robot-assisted group is non-inferior with respect to the control group. In general, it is not surprising to observe little to no difference between conventional and robot-assisted therapy in the context of studies where therapy dose and the therapy exercises/movements are designed to be similar, qualitatively and/or quantitatively, between groups. In this context, the haptic device primarily supports the therapist, providing additional motivation for the subjects to train, and delivering objective readouts (e.g., based on task performance, or kinematic and kinetic data) that can be used for monitoring, difficulty adaptation, or research purposes [29]. Nevertheless, the fact that a session of conventional therapy could be replaced without affecting the overall rehabilitation outcome opens promising avenues for further developing robotassisted therapy programs.

\section{Neurocognitive hand rehabilitation led to improvements on motor, sensory and cognitive scales}

Secondary outcome measures further support the equivalence analysis. After 4 weeks of treatment (T1), in addition to motor deficits, also sensory and cognitive deficits were concurrently reduced in both groups, with improvements in all the secondary clinical scales (i.e., proximal and distal arm impairment, functional ability, somatosensation, executive functions and cognitive 
control). The decrease in upper limb impairment (FMAUE, RG + 7.14 pts., CG + 6.85 pts) was clinically meaningful in both groups, and favorably compares to other work focusing on robot-assisted hand rehabilitation in subacute stroke, where changes between 3.0 to 5.3 FMA-UE points were typically reported [9]. Improvements were retained over time up to 7 months after the end of treatment. No significant differences were found between the groups in terms of changes with respect to baseline for all outcome measures. Only the BBT showed a significant effect of time, with additional increases in changes with respect to baseline after completion of the intervention (i.e, above $98 \%$ at T3). FMA$\mathrm{WH}$ also showed steady improvements over time after the end of the intervention (i.e. after T1), but these were not significant after Bonferroni correction. These further increases in BBT and FMA-WH suggest improvements in unilateral gross manual dexterity, which represents an essential element in the interaction with objects. This supports the approach of, whenever possible, focusing therapy on hand function training rather than proximal arm segments only, as distal training may promote impairment reduction in the entire arm [13, 14, 56].

Only minor improvements were observed in both groups over time in cognitive functions (i.e., FAB, MMSE, Albert Test), somatosensory function (i.e., EmNSA), and muscle tone control (i.e., MAS). These changes were small mostly due to the saturation of these scales in a mildly/moderately impaired population, and did not show significant changes between the groups and over time following T1. A decrease in MAS was observed in the CG, but not in the RG where a small, clinically non-relevant increase was observed. This is partly in line with a recent review [1], which analyzed changes in MAS of the paretic arm in 13 dose-matched RCTs and found negative effects on muscle tone reduction (i.e., increase in MAS) following robot-assisted therapy and a significant difference in favor of the respective control groups. This could possibly be caused by higher forces/muscle recruitment involved in robotassisted exercises, but was not monitored in the present study. Also, it is debatable whether a minor, temporary, increase in muscle tone would negatively affect functional recovery in subjects with stroke $[17,57]$. In the present study, the increase in MAS disappeared in follow-up assessments, and the different behavior of the two groups could also be explained by slightly higher baseline MAS in the CG. Additional studies are necessary to investigate how muscle tone evolves depending on subject conditions (e.g., lesion type), therapy type and intensity.

Finally, given the majority of hemorrhagic stroke survivors, a better functional recovery could have been expected in the control group compared to the robot- assisted group [58, 59]. Our results do not support this hypothesis, probably due to the rather mild impairment level of a majority of patients across both groups, indicating smaller lesions independent of the lesion type.

\section{Study limitations}

The participants involved in both groups were mostly mildly or moderately impaired (initial FMA $>29$ ) [43]. This led to ceiling/floor effects in some of the clinical sensory and motor assessments, which might have masked some of the intervention effects. This was, however, not imposed by our study design, subject screening or inclusion criteria, as the feasibility of the proposed robot-assisted therapy approach was also demonstrated in more severely impaired outpatients in the chronic stage after stroke [28]. No measure of real world upper limb use was included in the study design, and it therefore remains to be explored whether the proposed therapy leads to improvements in upper limb use in daily life. While the robot-assisted therapy program could be well integrated into a subacute rehabilitation program to complement the existing therapy, only patients with mild to moderate cognitive impairment were eligible to participate, as the intense therapy program challenged some patient's cognitive abilities. This did not allow to verify up to which cognitive impairment level the proposed approach could be applied, and the included patients only had little room for cognitive recovery. However, this was not the objective of this study since both groups received the same type of treatment. As additional possible confounder, all participants received additional conventional therapy sessions as part of their standard inpatient therapy program in parallel to the intervention, which could not be entirely substituted for ethical concerns. Nevertheless, we did achieve $15 \times 45$ min sessions over 4 weeks, which is comparable to other clinical trials or pilot studies on robot-assisted rehabilitation of hand function [51, 53, 54]. Furthermore, the results of this study are limited by the rather small sample size and should be interpreted with respect to the provided therapy and dose level. Finally, as is the case for any clinical trial at the subacute stage post stroke and of comparable sample size, the contribution of spontaneous recovery cannot be disentangled from intervention-induced recovery.

\section{Potential of neurocognitive robot-assisted rehabilitation of hand function}

The therapy intensity delivered in this study typically exceeded the amount of movement practice reported in the literature for conventional physio- or occupational therapy sessions (1.45 rep/min vs $0.92 \mathrm{rep} / \mathrm{min}$ ) [60]. Still, compared to the knowledge gained from animal studies and to recent high-dose clinical studies [61-63], 
this intensity might not be sufficient. It is important to note, however, that one "repetition" using the neurocognitive approach is not directly comparable to, e.g., reaching movements as typically reported in the literature. One repetition corresponds to one complex task (e.g., sponge identification) involving several actual movements, as well as sensory processing and cognition, demanding time, effort and concentration. An open question is whether delivering high (and potentially even higher) intensity of conventional therapy would be feasible in daily practice over several weeks, outside of a research study.

Establishing the non-inferiority in impairment reduction via robot-assisted therapy at a clinically-applicable dose is an important step towards opening new research avenues. While not all components of object manipulation (e.g., texture discrimination) can be trained with our robot, the proposed robot-assisted therapy of hand function, including sensorimotor and cognitive training, could ideally complement conventional therapy programs. Our therapy approach could further help increase the therapy dose provided to neurological patients, with the aim to positively impact functional recovery $[64,65]$ with only minimal additional burden on clinical staff. This could be achieved, after appropriate adjustments to the proposed technology, through semi-supervised therapy of multiple patients in parallel [66], minimallysupervised therapy during inpatients' spare time, or even a continuation thereof at home, as proposed in several promising pilot studies with passive devices [67, 68]. Especially regarding the latter, we find it crucial to introduce patients to such technology at an early stage during therapist supervision, which we here (and others) have shown to be feasible. In that sense, the results of this study demonstrating that neurocognitive robot-assisted therapy is also safe and well-accepted are a positive and necessary first step.

To reach the goal of minimally-supervised robotassisted rehabilitation, special attention should be devoted to the evaluation of usability and acceptance of rehabilitation devices, and in that sense, simple endeffector devices, such as the ReHapticKnob device used in this study, may be advantageous over upper limb exoskeletons often requiring long setup time and adjustments [9]. To meet user expectations and improve technology acceptance with respect to the current setup, more attention should be devoted to the design of ergonomic handles and to the adaptation of the difficulty levels of the exercises. Embedded clinical "intelligence" building on online robotic assessments, or performance metrics extracted from therapy sessions, should be further developed to provide means of accurately monitoring subjects' ability level and the evolution of their performance during treatment (or even after discharge), possibly adapting exercise difficulty autonomously to constantly challenge the user at an appropriate level [29]. Overall, such technology could help to increase the therapy dose subjects with stroke receive at the different stages of their rehabilitation, offer alternative solutions to enable earlier discharge of the subjects from the clinics, and provide continued and individually modulated therapy in the home environment after discharge.

\section{Conclusion}

This paper presents the results of a RCT investigating the equivalence in motor recovery between dosematched robot-assisted and conventional neurocognitive therapy of hand function after stroke. The results show that neurocognitive robot-assisted therapy can be well integrated in the clinical routine and allows for a noninferior motor recovery compared to conventional dosematched neurocognitive therapy when performed during inpatient rehabilitation in the subacute stage after stroke. An early familiarization of subjects with stroke to the use of such patient-tailored robot-assisted therapy program opens the doors to the use of such technology with minimal therapist supervision in the clinic, or directly at home after hospital discharge, to help increase the dose of hand therapy for persons with stroke.

\section{Abbreviations \\ FMA-UE: Fugl-meyer assessment of the upper extremity; GCP: Good clinical practice; RCT: Randomized controlled trial; RG: Robot-assisted group; \\ CG: Control group; NIHSS: National institutes of health stroke scale; LCF- R: Levels of cognitive functioning-revised; VASp: Visual analogue scale for pain; FMA-WH: Fugl-meyer assessment of wrist and hand; FMA-SE: Fugl- meyer assessment of shoulder and elbow; BBT: Box and blocks test; MAS: Modified ashworth scale; EmNSA: Erasmus MC Nottingham sensory assessment; MMSE: Mini mental state examination; AT: Albert test; FAB: Frontal assessment battery; MITT: Modified intention to treat}

\section{Acknowledgements}

The authors would like to thank Jürg Hartmann for handling part of the data collection, Dr. Gianni Rossi for his support throughout the study, Maya Kamber for her support with ethics and data monitoring, all the physiotherapists, speech therapists and neuro-psychologists from the Clinica Hildebrand that were involved in the trial execution, Mike D. Rinderknecht for his MATLAB toolboxes, as well as Janine Burren for her help with statistical analyses.

\section{Authors' contributions}

$J M, O L, F C$ and RG defined the study protocol. RR, OL and RG performed the data analysis and wrote the manuscript. JM implemented the robot-assisted therapy. JM, OL and RR coordinated the data collection and conducted the robot-assisted therapy sessions with the patients. AC and SR organized the conventional neurocognitive therapy and assisted the robotic therapy sessions with the patients. DD, CP, PR, FC helped with the study design and clinical implementation and were responsible for patient recruitment and screening. All authors read and approved the final manuscript. Raffaele Ranzani and Olivier Lambercy contributed equally to this work.

\section{Funding}

This work was supported by the National Center of Competence in Research on Neural Plasticity and Repair of the Swiss National Science Foundation (NCCR Neuro), the ETH CHIRP1 Research Grant on Cortically-Driven Assistance Adaptation during Sensorimotor Training, the Olga Mayenfisch Stiftung, the 
ETH Zurich Foundation in collaboration with Hocoma AG, and the Clinica Hildebrand Centro di Riabilitazione Brissago, Switzerland.

\section{Availability of data and materials}

The data supporting the conclusions of this article are included within the article.

\section{Ethics approval and consent to participate}

The study followed GCP-guidelines and was approved by the local Cantonal Ethics Committee Ticino (CE-TI: EC 2646) and Swissmedic (2013-MD-0002). All subjects gave written informed consent in accordance with the declaration of Helsinki.

\section{Consent for publication}

The individual in Fig. 1 consented to the publication of the photograph.

\section{Competing interests}

The authors declare that they have no competing interests.

\section{Author details}

${ }^{1}$ Rehabilitation Engineering Laboratory, Institute of Robotics and Intelligent Systems, Department of Health Sciences and Technology, BAA Lengghalde 5, 8008 Zurich, Switzerland. ${ }^{2}$ Clinica Hildebrand Centro di Riabilitazione Brissago, Via Crodolo, 6614 Brissago, Switzerland.

Received: 2 April 2020 Accepted: 11 August 2020

\section{Published online: 24 August 2020}

\section{References}

1. Veerbeek JM, Langbroek-Amersfoort AC, van Wegen EE, Meskers CG, Kwakkel G. Effects of robot-assisted therapy for the upper limb after stroke: a systematic review and meta-analysis. Neurorehabil Neural Repair. 2017; 31(2):107-21.

2. Klamroth-Marganska V, Blanco J, Campen K, Curt A, Dietz V, Ettlin T, et al. Three-dimensional, task-specific robot therapy of the arm after stroke: a multicentre, parallel-group randomised trial. Lancet Neurol. 2014;13(2):15966

3. Lo AC, Guarino PD, Richards LG, Haselkorn JK, Wittenberg GF, Federman DG, et al. Robot-assisted therapy for long-term upper-limb impairment after stroke. N Engl J Med. 2010;362(19):1772-83.

4. Maciejasz P, Eschweiler J, Gerlach-Hahn K, Jansen-Troy A, Leonhardt S. A survey on robotic devices for upper limb rehabilitation. J Neuroeng Rehabil. 2014;11(1):3

5. Lum PS, Burgar CG, Van der Loos M, Shor PC. MIME robotic device for upper-limb neurorehabilitation in subacute stroke subjects: a follow-up study. J Rehabil Res Dev. 2006;43(5):631.

6. Burgar CG, Garber SL, Van der Loos PhD HM, Deborah Kenney MSO. Robotassisted upper-limb therapy in acute rehabilitation setting following stroke: Department of Veterans Affairs multisite clinical trial. J Rehabil Res Dev. 2011:48(4):445

7. Masiero S, Armani M. Upper-limb robot-assisted therapy in rehabilitation of acute stroke patients: focused review and results of new randomized controlled trial. J Rehabil Res Dev. 2011:48(4):355.

8. Mehrholz J, Pohl M, Platz T, Kugler J, Elsner B. Electromechanical and robotassisted arm training for improving activities of daily living, arm function, and arm muscle strength after stroke. Cochrane Database Syst Rev. 2015; (11):CD006876. https://doi.org/10.1002/14651858.CD006876.pub4.

9. Lambercy O, Ranzani R, Gassert R. Robot-assisted rehabilitation of hand function. In: Rehabilitation Robotics: Elsevier. United States: Academic Press; 2018. p. 205-25.

10. Rodgers $\mathrm{H}$, Bosomworth $\mathrm{H}$, Krebs $\mathrm{HI}$, van Wijck F, Howel D, Wilson $\mathrm{N}$, et al. Robot assisted training for the upper limb after stroke (RATULS): a multicentre randomised controlled trial. Lancet. 2019.

11. Raghavan $P$. The nature of hand motor impairment after stroke and its treatment. Curr Treat Options Cardiovasc Med. 2007;9(3):221-8.

12. Fischer HC, Stubblefield K, Kline T, Luo X, Kenyon RV, Kamper DG. Hand rehabilitation following stroke: a pilot study of assisted finger extension training in a virtual environment. Top Stroke Rehabil. 2007;14(1):1-12.

13. Lambercy O, Dovat L, Yun H, Wee SK, Kuah CW, Chua KS, et al. Effects of a robot-assisted training of grasp and pronation/supination in chronic stroke: a pilot study. J Neuroeng Rehabil. 2011;8(1):63.
14. Hsieh YW, Lin KC, Wu CY, Shih TY, Li MW, Chen CL. Comparison of proximal versus distal upper-limb robotic rehabilitation on motor performance after stroke: a cluster controlled trial. Sci Rep. 2018;8(1):2091.

15. Lambercy O, Dovat L, Gassert R, Burdet E, Teo CL, Milner T. A haptic knob for rehabilitation of hand function. IEEE Trans Neural Syst Rehabil Eng. 2007; 15(3):356-66.

16. Lum PS, Godfrey SB, Brokaw EB, Holley RJ, Nichols D. Robotic approaches for rehabilitation of hand function after stroke. Am J Phys Med rehabil. 2012;91(11):S242-S54

17. Perfetti C, Grimaldi L. Rieducazione motoria dell'emiplegico. Italy: Ghedimedia; 1979.

18. Sallés $L$, Martín-Casas $P$, Gironès $X$, Durà MJ, Lafuente JV, Perfetti C. A neurocognitive approach for recovering upper extremity movement following subacute stroke: a randomized controlled pilot study. J Phys Ther Sci. 2017:29(4):665-72.

19. McEwen SE, Huijbregts MP, Ryan JD, Polatajko HJ. Cognitive strategy use to enhance motor skill acquisition post-stroke: a critical review. Brain Inj. 2009; 23(4):263-77.

20. Turville M, Carey LM, Matyas TA, Blennerhassett J. Change in functional arm use is associated with somatosensory skills after sensory retraining poststroke. Am J Occup Ther. 2017:71(3):7103190070p1-9.

21. Van de Winckel A, Wenderoth N, De Weerdt W, Sunaert S, Peeters R, Van Hecke W, et al. Frontoparietal involvement in passively guided shape and length discrimination: a comparison between subcortical stroke patients and healthy controls. Exp Brain Res Springer. 2012;220(2):179-89.

22. Lee $\mathrm{S}$, Bae $\mathrm{S}$, Jeon D, Kim KY. The effects of cognitive exercise therapy on chronic stroke patients' upper limb functions, activities of daily living and quality of life. J Phys Ther Sci. 2015;27(9):2787-91.

23. Carey $L$, Macdonell R, Matyas TA. SENSe: study of the effectiveness of Neurorehabilitation on sensation: a randomized controlled trial. Neurorehabil Neural Repair. 2011;25(4):304-13

24. Chanubol R, Wongphaet P, Chavanich N, Werner C, Hesse S, Bardeleben A et al. A randomized controlled trial of cognitive sensory motor training therapy on the recovery of arm function in acute stroke patients. Clin Rehabil. 2012;26(12):1096-104.

25. Morreale M, Marchione P, Pili A, Lauta A, Castiglia SF, Spallone A, et al. Early versus delayed rehabilitation treatment in hemiplegic patients with ischemic stroke: proprioceptive or cognitive approach. Eur J Phys Rehabil Med. 2016;52:81-9.

26. Albiol-Pérez S, Palacios-Navarro G, Guerrón-Paredes N, Gil-Gómez J-A, Quilis J-AL, Gil-Gómez H, et al. The Perfetti method, a novel virtual fine motor rehabilitation system for chronic acquired brain injury. In: Proceedings of the 8th International Conference on Pervasive Computing Technologies for Healthcare. Belgium: ICST (Institute for Computer Sciences, SocialInformatics and Telecommunications Engineering); 2014

27. Yu N, Wang K, Liu J. Dexterous haptic interaction for functional rehabilitation and assessment of the upper limb. In: Robotics and Biomimetics (ROBIO), 2014 IEEE International Conference on. Indonesia: IEEE; 2014.

28. Metzger J-C, Lambercy O, Califfi A, Conti FM, Gassert R. Neurocognitive robot-assisted therapy of hand function. IEEE Trans Haptics. 2014;7(2):140-9.

29. Metzger J-C, Lambercy O, Califfi A, Dinacci D, Petrillo C, Rossi P, et al. Assessment-driven selection and adaptation of exercise difficulty in robotassisted therapy: a pilot study with a hand rehabilitation robot. J Neuroeng Rehabil. 2014:11(1):154

30. Brott T, Adams HP, Olinger CP, Marler JR, Barsan WG, Biller J, et al. Measurements of acute cerebral infarction: a clinical examination scale. Stroke. 1989;20(7):864-70.

31. Huber W, Klingenberg G, Poeck K, Willmes K. Die supplemente zum Aachener aphasie test, aufbau und resultate der validierung. Neurolinguistik 1993;7(1):43-66

32. Hagen C. Rancho-los amigos levels of cognitive functioning-revised. In: TB| Rehabilitation Managed Care Environment. San Antonio, TX: An Interdisciplinary Approach to Rehabilitation Continuing Education Programs of America; 2000.

33. Fugl-Meyer AR, Jääskö L, Leyman I, Olsson S, Steglind S. The post-stroke hemiplegic patient. 1. A method for evaluation of physical performance. Scand J Rehabil Med. 1975:7(1):13-31.

34. Mathiowetz V, Volland G, Kashman N, Weber K. Adult norms for the box and block test of manual dexterity. Am J Occup Ther. 1985:39(6): 386-91. 
35. Charalambous CP. Interrater reliability of a modified Ashworth scale of muscle spasticity. In: Classic Papers in Orthopaedics. UK: Springer; 2014. p. $415-7$.

36. Stolk-Hornsveld F, Crow J, Hendriks E, Van Der Baan R. Harmeling-Van der Wel B. the Erasmus MC modifications to the (revised) Nottingham sensory assessment: a reliable somatosensory assessment measure for patients with intracranial disorders. Clin Rehabil. 2006;20(2):160-72.

37. Folstein MF, Folstein SE, McHugh PR. "Mini-mental state": a practical method for grading the cognitive state of patients for the clinician. J Psychiatr Res. 1975:12(3):189-98.

38. Albert ML. A simple test of visual neglect. Neurology. 1973.

39. Dubois B, Slachevsky A, Litvan I, Pillon B. The FAB a frontal assessment battery at bedside. Neurology. 2000;55(11):1621-6.

40. Walker $E$, Nowacki AS. Understanding equivalence and noninferiority testing. J Gen Intern Med. 2011;26(2):192-6.

41. Wagner JM, Rhodes JA, Patten C. Reproducibility and minimal detectable change of three-dimensional kinematic analysis of reaching tasks in people with hemiparesis after stroke. Phys Ther. 2008;88(5):652-63.

42. D'Agostino RB, Sullivan LM, Beiser AS. Introductory applied biostatistics. Australia: Thomson brooks/Cole; 2006.

43. Woytowicz EJ, Rietschel JC, Goodman RN, Conroy SS, Sorkin JD, Whitall J, et al. Determining levels of upper extremity movement impairment by applying a cluster analysis to the Fugl-Meyer assessment of the upper extremity in chronic stroke. Arch Phys Med Rehab. 2017;98(3):456-62.

44. Shelton FN, Volpe BT, Reding M. Motor impairment as a predictor of functional recovery and guide to rehabilitation treatment after stroke. Neurorehabil Neural Repair. 2001;15(3):229-37.

45. Chen H-M, Chen CC, Hsueh I-P, Huang S-L, Hsieh C-L. Test-retest reproducibility and smallest real difference of 5 hand function tests in patients with stroke. Neurorehabil Neural Repair. 2009;23(5):435-40.

46. Shaw L, Rodgers H, Price C, van Wijck F, Shackley P, Steen N, et al. BoTULS: a multicentre randomised controlled trial to evaluate the clinical effectiveness and cost-effectiveness of treating upper limb spasticity due to stroke with botulinum toxin type a. Health Technol Assess. 2010;14(26):1-113.

47. Feeney J, Savva GM, O'Regan C, King-Kallimanis B, Cronin H, Kenny RA. Measurement error, reliability, and minimum detectable change in the minimental state examination, Montreal cognitive assessment, and color trails test among community living middle-aged and older adults. J Alzheimers Dis. 2016;53(3):1107-14.

48. Rowe JB, Chan V, Ingemanson ML, Cramer SC, Wolbrecht ET, Reinkensmeyer DJ. Robotic assistance for training finger movement using a Hebbian model: a randomized controlled trial. Neurorehabil Neural Repair. 2017;31(8): 769-80.

49. Connelly L, Jia Y, Toro ML, Stoykov ME, Kenyon RV, Kamper DG. A pneumatic glove and immersive virtual reality environment for hand rehabilitative training after stroke. IEEE Trans Neural Syst Rehabil Eng. 2010; 18(5):551-9.

50. Thielbar KO, Triandafilou KM, Fischer HC, OToole JM, Corrigan ML, Ochoa $\mathrm{JM}$, et al. Benefits of using a voice and EMG-driven actuated glove to support occupational therapy for stroke survivors. IEEE Trans Neural Syst Rehabil Eng. 2017;25(3):297-305.

51. Susanto EA, Tong RK, Ockenfeld C, Ho NS. Efficacy of robot-assisted fingers training in chronic stroke survivors: a pilot randomized-controlled trial. J Neuroeng Rehabil. 2015;12(1):42.

52. Kutner NG, Zhang R, Butler AJ, Wolf SL, Alberts JL. Quality-of-life change associated with robotic-assisted therapy to improve hand motor function in patients with subacute stroke: a randomized clinical trial. Phys Ther. 2010; 90(4):493-504

53. Hwang $\mathrm{CH}$, Seong JW, Son D-S. Individual finger synchronized robotassisted hand rehabilitation in subacute to chronic stroke: a prospective randomized clinical trial of efficacy. Clin Rehabil. 2012;26(8):696-704.

54. Vanoglio F, Bernocchi P, Mulè C, Garofali F, Mora C, Taveggia G, et al. Feasibility and efficacy of a robotic device for hand rehabilitation in hemiplegic stroke patients: a randomized pilot controlled study. Clin Rehabil. 2017;31(3):351-60.

55. Orihuela-Espina F, Roldán GF, Sánchez-Villavicencio I, Palafox L, Leder R, Sucar $L E$, et al. Robot training for hand motor recovery in subacute stroke patients: a randomized controlled trial. J Hand Ther. 2016;29(1):51-7.

56. Stein J, Bishop L, Gillen G, Helbok R. Robot-assisted exercise for hand weakness after stroke: a pilot study. Am J Phys Med Rehabil. 2011; 90(11):887-94.
57. Shumway-Cook A, Woollacott MH. Motor control: translating research into clinical practice. United States: Lippincott Williams \& Wilkins; 2007.

58. Paolucci S, Antonucci G, Grasso MG, Bragoni M, Coiro P, De Angelis D, et al. Functional outcome of ischemic and hemorrhagic stroke patients after inpatient rehabilitation: a matched comparison. Stroke. 2003;34(12):2861-5.

59. Kelly PJ, Furie KL, Shafqat S, Rallis N, Chang Y, JJAopm S, et al. Functional recovery following rehabilitation after hemorrhagic and ischemic stroke. Arch Phys Med Rehabil. 2003;84(7):968-72.

60. Lang CE, MacDonald JR, Reisman DS, Boyd L, Kimberley TJ, Schindler-Ivens SM, et al. Observation of amounts of movement practice provided during stroke rehabilitation. Arch Phys Med Rehab. 2009;90(10):1692-8.

61. Ward NS, Brander F, KJJNNP K. Intensive upper limb neurorehabilitation in chronic stroke: outcomes from the Queen Square programme. J Neurol Neurosurg Psych. 2019;90(5):498-506.

62. McCabe J, Monkiewicz M, Holcomb J, Pundik S, Daly JJ. Comparison of robotics, functional electrical stimulation, and motor learning methods for treatment of persistent upper extremity dysfunction after stroke: a randomized controlled trial. Arch Phys Med Rehabil. 2015;96(6):981-90.

63. Daly JJ, MCCabe JP, Holcomb J, Monkiewicz M, Gansen J, SJN P, et al. LongDose intensive therapy is necessary for strong, clinically significant, upper limb functional gains and retained gains in Severe/Moderate chronic stroke. Neurorehabil Neural Repair. 2019;33(7):523-37.

64. Han C, Wang Q. Meng P-p, qi M-z. effects of intensity of arm training on hemiplegic upper extremity motor recovery in stroke patients: a randomized controlled trial. Clin Rehabil. 2013;27(1):75-81.

65. Veerbeek JM, van Wegen $E$, van Peppen $R$, van der Wees PJ, Hendriks E, Rietberg $\mathrm{M}$, et al. What is the evidence for physical therapy poststroke? A systematic review and meta-analysis. PLoS One. 2014;9(2):e87987.

66. Busching I, Sehle A, Sturner J, Liepert J. Using an upper extremity exoskeleton for semi-autonomous exercise during inpatient neurological rehabilitation- a pilot study. J Neuroeng Rehabil. 2018;15.

67. Hayward KS, Neibling BA, Barker RN. Self-administered, home-based SMART (sensorimotor active rehabilitation training) arm training: a single-case report. Am J Occup Ther. 2015;69(4):6904210020p1-8.

68. Amirabdollahian F, Ates S, Basteris A, Cesario A, Buurke J, Hermens H, et al. Design, development and deployment of a hand/wrist exoskeleton for home-based rehabilitation after stroke-SCRIPT project. Robotica. 2014;32(8): 1331-46.

\section{Publisher's Note}

Springer Nature remains neutral with regard to jurisdictional claims in published maps and institutional affiliations.
Ready to submit your research? Choose BMC and benefit from:
- fast, convenient online submission
- thorough peer review by experienced researchers in your field
- rapid publication on acceptance
- support for research data, including large and complex data types
- gold Open Access which fosters wider collaboration and increased citations
- maximum visibility for your research: over $100 \mathrm{M}$ website views per year
At BMC, research is always in progress. 